\title{
To predict and understand anal fissure healing
}

\author{
Mariusz Madalinski
}

Accepted: 28 May 2010 / Published online: 12 June 2010

(C) Springer-Verlag 2010

\section{Dear Editor:}

Lateral internal sphincterotomy is called the gold standard for chronic anal fissure (CAF), but chemical sphincterotomy (CS) has been recognized by many doctors as the first line of treatment for CAF, due to a risk of gas and stool incontinence after surgery. CS is so important for cost therapy too.

I am pleased to read that authors Gil $\mathrm{J}$ et al. gave so much attention to my theory of anal fissure healing and a meaning of anal sphincters "strechability". Their study also confirmed postulated by me that reduction of the mean anal pressure (commonly examined) is no prerequisite for CAF healing and that we should look for other predictable factors. Therefore I would like to appreciate a meaning of Gil J et al.'s ratio resting/voluntary contraction pressure.
Much as we would like to see it confirmed in other studies, I am afraid that we need to understand much more than we suppose. Botulinum toxin (TOX) as other medication shows a therapeutic effect related to the dose. TOX also shows an effect related to its dilution. Probably also the optimal angle for needle should be considered, during injection of TOX. Moreover, TOX improves wound healing also outside the anus where blood supply is not limited by sphincters. Therefore we should think that a place of TOX injection (its distance from an anal fissure) may be relevant. I hope that other researches confirm the results of Gil et al., but we should always take a higher dose of TOX into account, because it was proved that TOX may be successful after glyceryl trinitrate treatment failures.
M. Madalinski

NHS Lothian, University Hospitals Division,

Edinburgh, UK

M. Madalinski $(\square)$

Gastroenterology Department, Western General Hospital,

Crewe Road South,

Edinburgh EH4 2XU, UK

e-mail: m.h.madalinski@pro.onet.pl 